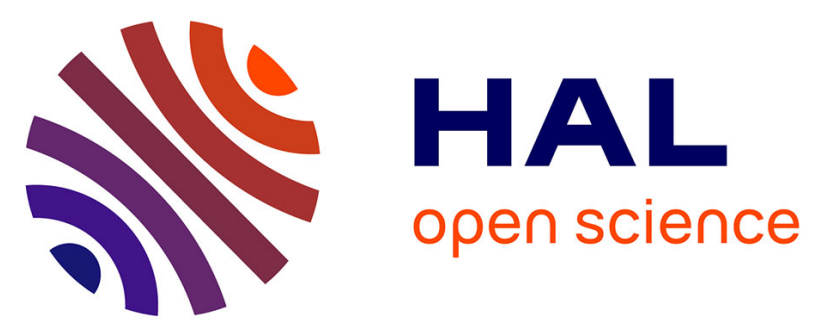

\title{
De la terre patrimoine à la terre ressource: tensions entre structures foncières héritées et nouvelles perspectives des acteurs paysans en Afrique de l'Est.
} Bernard Charlery de La Masselière, Sylvain Racaud

\section{- To cite this version:}

Bernard Charlery de La Masselière, Sylvain Racaud. De la terre patrimoine à la terre ressource: tensions entre structures foncières héritées et nouvelles perspectives des acteurs paysans en Afrique de l'Est.. Bulletin de l'Association de géographes français, 2012, 89 (3), pp.412-426. 10.3406/bagf.2012.8280 . hal-02326211

\section{HAL Id: hal-02326211 \\ https://hal.science/hal-02326211}

Submitted on 22 Oct 2019

HAL is a multi-disciplinary open access archive for the deposit and dissemination of scientific research documents, whether they are published or not. The documents may come from teaching and research institutions in France or abroad, or from public or private research centers.
L'archive ouverte pluridisciplinaire HAL, est destinée au dépôt et à la diffusion de documents scientifiques de niveau recherche, publiés ou non, émanant des établissements d'enseignement et de recherche français ou étrangers, des laboratoires publics ou privés. 


\section{per \\ s}

Bulletin de l'Association de géographes français

\section{De la terre patrimoine à la terre ressource : tensions entre} structures foncières héritées et nouvelles perspectives des acteurs paysans en Afrique de l'Est. (From heritage land to resource land: tensions between inherited land structures and new perspectives of peasant stakeholders in Eastern Africa) Bernard Charlery de La Masselière, Sylvain Racaud

\section{Citer ce document / Cite this document :}

Charlery de La Masselière Bernard, Racaud Sylvain. De la terre patrimoine à la terre ressource : tensions entre structures foncières héritées et nouvelles perspectives des acteurs paysans en Afrique de l'Est. (From heritage land to resource land: tensions between inherited land structures and new perspectives of peasant stakeholders in Eastern Africa). In: Bulletin de I'Association de géographes français, 89e année, 2012-3. Terres et tensions en Afrique. pp. 412-426;

doi : https://doi.org/10.3406/bagf.2012.8280

https://www.persee.fr/doc/bagf_0004-5322_2012_num_89_3_8280

Fichier pdf généré le 26/04/2018 


\begin{abstract}
There are strong links between the external and internal dimensions of the land issue which, moreover, deals with many stakes : social, identity, political, economic and environmental. Interactions of scale, of time or space, add further to this complexity, giving rise to heterogeneous situations which are sources of conflicts. In East Africa, the agricultural modernization has been imposed on local systems following a process of territorial construction which has gradually developed into a rigid and heterogeneous framework; the land tenure blockage gave it sometimes a dramatic dimension. Under this process redefining the territories of production and the local identities, rural-based societies are suffering from historical rigidities and are handicapped by the extreme fragmentation and compartmentalization of production units. The attempts made by the producers to be involved in promising markets or in often uncertain agricultural chains redefine a composite and unequal "area of resources", where access to land, to water, to credit, to infrastructures and to information networks is a new comparative advantage. The objective of this paper is to explore some new ideas from specific studies which has been carried out on East African mountains, on the assumption that the diversity and the heterogeneity of the new socio-economical and territorial logics are the sign of the search of a new horizon of expectation, which could better articulate the moral economy of local societies with a project which could offer access to a larger citizenship
\end{abstract}

\title{
Résumé
}

Il existe des liens forts entre les dimensions externes et internes de la question foncière qui, par ailleurs, porte en elle des enjeux multiples : sociaux, identitaires, politiques, économiques et environnementaux. A cette complexité s'ajoutent des interactions d'échelles, de temps et d'espaces, qui créent des situations très hétérogènes et sources de conflits. En Afrique de l'Est, la modernisation agricole s 'est imposée aux systèmes locaux suivant un processus de construction territoriale qui s 'est peu à peu figé en un cadre rigide et hétérogène, auquel le blocage foncier a donné une dimension parfois dramatique. Dans ce processus de redéfinition des territoires de production et de redéfinition des identités, les sociétés à base rurale pâtissent de rigidités historiques et se trouvent handicapées par l'extrême fragmentation et le cloisonnement des unités de production. Les tentatives d'engagement des producteurs dans les marchés porteurs ou dans des filières souvent incertaines redéfinissent un «espace de ressources » composite et inégal, où I 'accès à la terre, à I 'eau, au crédit, aux voies de communication et aux circuits d'information constitue un nouvel avantage comparatif. $L$ 'objectif de cet article est d'ouvrir quelques pistes de réflexion à partir de travaux ponctuels menés sur les montagnes d'Afrique de l'Est, selon l'hypothèse principale que la démultiplication et l'hétérogénéité de nouvelles logiques socio-économiques et territoriales sont le signe de la recherche d'un nouvel horizon d'attente, permettant d'articuler plus efficacement l'économie morale des sociétés locales à un projet de société qui offrirait l'accès à une citoyenneté plus large.

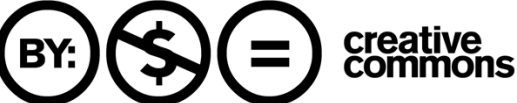




\title{
De la terre patrimoine à la terre ressource : tensions entre structures foncières héritées et nouvelles perspectives des acteurs paysans en Afrique de l'Est.
}

\author{
(FROM HERITAGE LAND TO RESOURCE LAND : TENSIONS \\ BETWEEN INHERITED LAND STRUCTURES AND NEW \\ PERSPECTIVES OF PEASANT STAKEHOLDERS IN EASTERN \\ AFRICA)
}

\section{Bernard CHARLERY de la MASSELIÈRE ${ }^{*}$ \& Sylvain RACAUD**}

\begin{abstract}
RÉSUMÉ - Il existe des liens forts entre les dimensions externes et internes de la question foncière qui, par ailleurs, porte en elle des enjeux multiples : sociaux, identitaires, politiques, économiques et environnementaux. A cette complexité s'ajoutent des interactions d'échelles, de temps et d'espaces, qui créent des situations très hétérogènes et sources de conflits. En Afrique de l'Est, la modernisation agricole $s$ 'est imposée aux systèmes locaux suivant un processus de construction territoriale qui $s$ 'est peu à peu figé en un cadre rigide et hétérogène, auquel le blocage foncier a donné une dimension parfois dramatique. Dans ce processus de redéfinition des territoires de production et de redéfinition des identités, les sociétés à base rurale pâtissent de rigidités historiques et se trouvent handicapées par l'extrême fragmentation et le cloisonnement des unités de production. Les tentatives d'engagement des producteurs dans les marchés porteurs ou dans des filières souvent incertaines redéfinissent un " espace de ressources" composite et inégal, où l'accès à la terre, à l'eau, au crédit, aux voies de communication et aux circuits d'information constitue un nouvel avantage comparatif. $L$ 'objectif de cet article est d'ouvrir quelques pistes de réflexion à partir de travaux ponctuels menés sur les montagnes d'Afrique de l'Est, selon l'hypothèse principale que la démultiplication et l'hétérogénéité de nouvelles logiques socioéconomiques et territoriales sont le signe de la recherche d'un nouvel horizon d'attente, permettant d'articuler plus efficacement l'économie morale des sociétés locales à un projet de société qui offrirait l'accès à une citoyenneté plus large.
\end{abstract}

\footnotetext{
* Professeur de géographie, UMR Dynamiques rurales, Université Toulouse2-Le Mirail Courriel : charlery@univ-tlse2.fr

* Doctorant, UMR Dynamiques rurales, Université Toulouse2-Le Mirail - Courriel : geosracaud@gmail.com
} 
Mots-clés : Territoire - Ressource - Conflits - Marchés - Mobilité - Génération Afrique de l'Est

ABSTRACT - There are strong links between the external and internal dimensions of the land issue which, moreover, deals with many stakes: social, identity, political, economic and environmental. Interactions of scale, of time or space, add further to this complexity, giving rise to heterogeneous situations which are sources of conflicts. In East Africa, the agricultural modernization has been imposed on local systems following a process of territorial construction which has gradually developed into a rigid and heterogeneous framework; the land tenure blockage gave it sometimes a dramatic dimension. Under this process redefining the territories of production and the local identities, rural-based societies are suffering from historical rigidities and are handicapped by the extreme fragmentation and compartmentalization of production units. The attempts made by the producers to be involved in promising markets or in often uncertain agricultural chains redefine a composite and unequal "area of resources", where access to land, to water, to credit, to infrastructures and to information networks is a new comparative advantage. The objective of this paper is to explore some new ideas from specific studies which has been carried out on East African mountains, on the assumption that the diversity and the heterogeneity of the new socio-economical and territorial logics are the sign of the search of a new horizon of expectation, which could better articulate the moral economy of local societies with a project which could offer access to a larger citizenship

Keywords: Territory - Resource - Conflicts - Markets - Mobility - Generation Eastern Africa

Les concessions de terres attribuées à des sociétés ou à des pays étrangers ont domné une dimension "globale » à l'acuité de la question foncière en Afrique. Les deux dernières décennies en avaient marqué le caractère identitaire à l'échelle nationale. Un dossier sur La course mondiale au foncier, paru en 2012, rappelle que «Les deux-tiers des terres acquises se situent en Afrique. La forte (pour ne pas dire uniforme) orientation Nord-Sud et la participation importante de gouvernements et d'entreprises publiques en tant qu'acheteurs évoquent l'appellation courante de nouveau colonialisme » [Wily 2012]. En 1960 déjà, au cours du séminaire de Léopoldville consacré aux systèmes agraires africains, Daniel Biebuyck soulignait que l'aliénation de grandes superficies de terres au profit de l'économie européenne et la création de réserves naturelles avaient fini par provoquer des pénuries artificielles de terre et suscité la suspicion et le sentiment d'insécurité chez les populations qui se sentaient directement menacées dans l'exercice de leurs droits et privilèges fonciers et politiques [Biebuyck 1963, p. 45]. Il existe donc des liens forts entre les dimensions externes et internes de la question foncière qui, par ailleurs, porte en elle des enjeux multiples : sociaux, identitaires, politiques, économiques, environnementaux etc. A cette complexité s'ajoutent des 
interactions d'échelles, de temps et d'espaces, qui créent des situations très hétérogènes et sources de conflits.

Sur les hautes terres d'Afrique de l'Est, le contexte est marqué par une raréfaction ancienne de la ressource en terre, élément parmi d'autres de la crise du modèle spatial produit par la logique du développement colonial. Déjà, à l'aube de la colonisation, l'Afrique de l'Est avait été confrontée à une remise en cause des structures sociales et territoriales de groupes, communautés ou sociétés très diversifiés ainsi que des rapports et des modes d'échange qu'ils entretenaient [Charlery de la Masselière 2001]. La politique coloniale a consisté à mettre en compatibilité le fonctionnement endogène des sociétés agraires et les orientations du marché, en utilisant les nécessités d'évolution, produites par les contradictions internes des sociétés indigènes. Agissant directement ou indirectement, le colonisateur s'est ainsi attaqué à tout ce qui pouvait cristalliser une appartenance multiple à un espace composite (compétences et droits administratifs, droits collectifs) et indéterminé (terres sans maitre ou faiblement appropriées). Le nouvel ordre territorial s'appuyait par là sur un double processus : d'effacement de la nature sociale et symbolique de l'espace, d'un côté ; d'encadrement territorial et d'intégration spatiale d'un autre, sur la base d'une surdétermination de l'espace par la rationalité technique. Les réserves forestières, les grands projets de développement, les grandes plantations (estates) et les fermes mixtes européennes ont encerclé les terroirs traditionnels, quand ils ne les ont pas expropriés. La modernisation agricole s'est ainsi imposée aux systèmes locaux suivant un processus de construction territoriale qui s'est peu à peu figé en un cadre rigide et hétérogène, auquel le blocage foncier a donné une dimension parfois dramatique. Depuis la fin des années 1980, la libéralisation économique associée à la croissance des villes moyennes mais aussi le développement du multipartisme et la diffusion des nouveaux modes de communication contribuent à ouvrir l'espace public et privé au sein duquel individus et groupes sociaux construisent leurs systèmes de ressources. Ils intègrent à d'autres aires de compétition les territoires de référence d'une idéologie ruralisante confrontée aux impasses d'une économie agrarienne trop exclusive. Dans ce processus de redéfinition des territoires de production et de redéfinition des identités, les sociétés à base rurale pâtissent de rigidités historiques et se trouvent handicapées par l'extrême fragmentation et le cloisonnement des unités de production, qui contribuent à individualiser les trajectoires multiples et conflictuelles par lesquelles chacun essaie de répondre aux nécessités du moment. On se trouve face à un véritable puzzle que les règles émises à un niveau plus général permettent difficilement de reconstituer. Les tentatives d'engagement des producteurs dans les marchés porteurs ou dans des filières souvent incertaines redéfinissent un espace «de ressources " composite et inégal, où l'accès à la terre, à l'eau, au crédit, aux voies de communication et aux circuits d'information constitue un nouvel avantage comparatif. 
Comme le soulignent les auteurs du rapport sur les résultats du projet de recherche CLAIMS", «les dimensions intrafamiliales et intergénérationnelles [...] sont pratiquement absentes des travaux développés ces dernières décennies sur la question foncière. L'allocation des ressources au sein des unités économiques de base a certes déjà été étudiée [...] mais, dans les faits, la question des droits sur la terre n'a guère été abordée... 》 [Chauveau \& al. 2006]. En Afrique de l'Est, on manque de données systématiques sur les transferts fonciers, l'économie rurale, l'évolution des unités de base (consommation, production, solidarité,...). L'objectif de cet article est donc d'ouvrir quelques pistes de réflexion à partir de travaux ponctuels menés sur les montagnes d'Afrique de l'Est', selon l'hypothèse principale que la démultiplication et l'hétérogénéité de nouvelles logiques socio-économiques et territoriales sont le signe de la recherche d'un nouvel horizon d'attente, permettant d'articuler plus efficacement l'économie morale des sociétés locales à un projet de société qui offrirait l'accès à une citoyenneté plus large.

\section{Tensions foncières et marchandisation de l'économie rurale dans les Uporoto Mountains ${ }^{3}$}

Les montagnes Uporotos se situent au sud-ouest de la Tanzanie, à proximité de la Zambie et du Malawi. L'ensemble géomorphologique abrite plus d'un million d'habitants dont environ 300000 sont rassemblés dans la ville de Mbeya, capitale éponyme de la région localisée à une altitude de 1700 mètres. Les densités rurales sont en moyenne de $138 \mathrm{~h} / \mathrm{Km}^{2}$. Anciennement arrimées dans des réseaux commerciaux de long cours par les filières thé, café et pyrèthre, les Uporotos sont le lieu d'une importante production agricole dont les modalités d'intégration au marché s'ajustent aux changements de l'environnement économique. L'économie urbaine locale et l'économie rurale sont considérablement imbriquées et dépendent du commerce des produits de la terre. Le moteur de la production agricole est la demande urbaine nationale, ces campagnes d'altitude fournissant en pommes de terre et en bananes le marché de Dar es Salaam et d'autres centres urbains secondaires. Les pommes de terre sont cultivées depuis longtemps dans la région, mais c'est à partir de la fin des années 1970 qu'elles font l'objet d'un commerce intense. Plus bas dans la montagne la culture de la banane, puis récemment celle de l'avocat s'imposent comme activités génératrices de revenus. La pénétration de

\footnotetext{
${ }_{1}^{1}$ Changes in Land Access, Institutions and Markets in West Africa

${ }^{2}$ Ces travaux sont menés depuis quelques années sur le Mont Elgon (Ouganda), le Mont Kenya, le Kilimandjaro et les Uporoto Mountains (Tanzanie), avec le soutien de l'IFRANairobi, de l'Union Européenne ( $7^{\circ} \mathrm{FP}$ SSH), du programme CORUS (MAE-France), des Maisons des Sciences de l'Homme de Toulouse et d'Aquitaine

${ }^{3}$ Cette partie s'appuie sur le travail de thèse de Sylvain Racaud, les données étant issues des enquêtes de terrain 2010 et 2011
} 
l'économie globale a conduit à une marchandisation de la vie rurale et des moyens de production que sont la force de travail, les intrants et la terre. Ce phénomène s'est accéléré depuis les années 1980 et demeure fonction des rythmes d'intégration au marché, différenciés selon les terroirs; il se traduit par une amplification des flux entre à la fois des espaces plus nombreux et des acteurs plus variés.

\subsection{La transformation des moyens de production au regard de la marchandisation de l'économie rurale}

Les modes coutumiers de gestion foncière ont été mis à mal par le développement de rapports marchands au sein de la société rurale en prise avec la globalité. Le tournant date des années 1980 lorsque les variétés commerciales ont été introduites (arika pour la pomme de terre) pour répondre à la demande urbaine de chips. L'accès au marché a été facilité par la venue de nombreux acteurs privés. La ressource en capital monétaire est devenu indispensable pour avoir accès aux intrants (semences, engrais, produits phytosanitaires) et mobiliser la main d'œuvre. Les fortes potentialités agricoles des Uporotos ont attiré de nombreux migrants, dont certains originaires du district voisin de Makete, désireux de s'impliquer dans l'agriculture que ce soit au niveau de la production ou de la commercialisation. Certains ont acheté des terres aux autorités locales.

Les modes d'acquisition du foncier restent traditionnels. La terre s'obtient par héritage aussi bien pour les garçons que pour les filles tant pour les Nyakyusa que pour les Safwa ${ }^{4}$. A cause de la forte croissance de la population, ${ }^{5}$ la taille des surfaces cultivables par ménage a diminué pour atteindre aujourd'hui 2,27 acres, soit 0,9 hectare $^{6}$. La création de réserves forestières (Poroto Ridge Forest Reserves, Rungwe Forest Reserve, Livingstone Forest Reserve pour les plus vastes) a accentué cette pression foncière. Cela a déjà engendré des conflits, parfois violents ${ }^{7}$. La parcellisation du patrimoine foncier a favorisé l'émigration de jeunes en particulier dans le district saturé de Rungwe. Dans la montagne, entre Mbeya et le mont Rungwe, la pression foncière se traduit par un épuisement des sols et par des

\footnotetext{
${ }^{4}$ Les Nyakyusa sont le groupe principal du Rungwe, les Safwa sont majoritaires dans la zone Uporoto, entre Mbeya town et le Rungwe. Mbeya town, fondée sur la terre Safwa est aujourd'hui principalement peuplée par les Nyakyusa.

5 2,8\%/an dans Mbeya rural, 1\%/an dans le Rungwe (foyer d'émigration très fortement saturé). Données sur la période 1988-2002, d'après le recensement de 2002. URT, Regional Commissioner's Office (2011) Mbeya Region Socio-economic Profile, draft version august 2011. 322p.

${ }^{6} 1$ acre est une superficie de $4000 \mathrm{~m}^{2}$, soit approximativement un carré de $63 \mathrm{~m}$ de côté. De fortes variations ont été observées : en moyenne les foyers disposent de moins de 1 acre à Simambwe, de 1 à 4 acres à Ibililo, 2 à 5 acres à Santilya.

${ }^{7}$ En 2009 un ranger a été tué aux alentours du village d'Igoma.
} 
phénomènes d'érosion dus à leur surexploitation et à l'abandon des techniques traditionnelles, en particulier la jachère et les terrasses [Sokoni 2001]. Il n'y a plus de réserves disponibles et la fertilité, sur laquelle s'était appuyé le développement de ces terroirs, est remise en cause. La surexploitation de la terre répond à la nécessité impérieuse de dégager du cash.

La valeur de la terre s'est fortement accrue. Les prix du foncier ont explosé en l'espace de quelques années : à Ibililo il y a dix ans une acre près du marché valait 500000 TZS (237 euros), aujourd'hui cette même acre vaut 3 millions de TZS (1 422 euros). L'effet " route » et le critère de proximité jouent à plein dans la fixation des prix : à Ntokela, le long de la seule route asphaltée qui traverse le massif, une acre est estimée à 2 millions de TZS alors que dans la zone reculée d'Umalila, son prix ne dépasse pas 400000 TZS. Cependant le marché de la terre reste limité : il concerne surtout de petites parcelles qui sont vendues prioritairement à des membres du village ou gens des alentours. Les entreprises de grande ampleur sont marginales : un homme d'affaire sudafricain a acheté et loué des champs dans le Rungwe pour planter une variété " améliorée » d'avocats destinés à l'exportation ; quelques businessmen de Dar es Salaam peuvent construire une maison ${ }^{8}$ dans le Rungwe et acheter ensuite des parcelles dispersées et de faibles dimensions (entre 1 et 4 acres) en s'appuyant sur un agent local. Ces investissements concernent les cultures pérennes comme la banane ou l'avocat et, à plus court terme par le biais de location de parcelles, les cultures à cycle court (pomme de terre, chou) dont les risques liés au climat sont plus élevés.

Les baux s'étalent sur une saison de récolte et leurs prix diffèrent en fonction du lieu. Dans les zones «retirées " comme Santilya située à environ de $30 \mathrm{Km}$ de la Tanzam ${ }^{9}$, la location d'une acre pour une saison vaut entre 16 000 et 25000 TZS ; le montant pour la même superficie à Ntokela peut dépasser 100000 TZS. Ces deux villages jouissent de conditions agroenvironnementales similaires et les cultures plantées sont identiques : c'est l'accessibilité au marché qui contribue à différencier le prix du foncier et des marchandises. Ce facteur intervient aussi dans la variété de la gamme des acteurs impliqués dans l'agriculture. La zone Umalila attire des acteurs de Mbalizi, ville située à $10 \mathrm{Km}$ à l'est de Mbeya et qui compte plus de 30000 habitants, qui louent des parcelles pour remédier à l'indisponibilité de terres au lieu de résidence. Ils plantent à la fois des cultures pour l'autoconsommation (maïs), du vivrier marchand (haricot) et des cultures commerciales (pomme de terre, chou et pyrèthre). Par contre les espaces de production à proximité de l'axe Mbeya-Malawi sont des lieux où s'exercent une forte confrontation entre les locaux et les businessmen en quête de marchandises. L'origine géographique de ces acteurs est plus variée : elle comprend des personnes

\footnotetext{
${ }^{8}$ Selon la nouvelle législation foncière de 1999 : le « Land Act » et le « Village Land Act ».

${ }^{9}$ Axe Dar es Salaam-Lusaka
} 
originaires de grands centres urbains tanzaniens comme Dar es Salaam, Dodoma, Iringa, etc. et évidemment Mbeya. Ces investisseurs sont en très forte compétition pour la location de terres en vue de produire principalement de la pomme de terre. Ils s'appuient sur des superviseurs locaux en charge de trouver les superficies et de gérer la production et parfois même la commercialisation.

Ces changements dans les rapports de production et le statut des moyens de production se réalisent dans un contexte de réduction de l'intervention de l'Etat, et de dérégulations économiques mises en place depuis 1986. Les relations entre des acteurs plus nombreux et variés à la fois au niveau local et plus global s'en sont trouvées amplifiées et complexifiées.

\subsection{Le foncier : une ressource en dernier ressort}

Dans les années 1970 l'Etat avait confié la gestion des terres vacantes aux communautés locales. De nos jours certains villages disposent encore de quelques réserves qu'ils mettent en location pour leurs habitants. Santilya possède une réserve de 300 acres dont la moitié est mise en valeur, tandis qu'Ibililo ne détient plus qu'une dizaine d'acres toutes mis en culture par le comité villageois. Les terrains ne sont pas titrés officiellement, le "savoir local » gérant le foncier et les éventuels conflits. Cependant, les populations interrogées répondent systématiquement «oui » quand on les interroge sur la possession d'un titre foncier : l'occupation détermine l'appropriation. Le Village Land Act et le Land Act de 1999 ont renforcé les rôles des conseils villageois et ont reconnu ces droits coutumiers. L'objectif affiché est d'assurer la sécurité foncière pour ensuite développer l'agriculture et les investissements. Chaque propriétaire est libre de céder sa terre. Le village est censé encaisser $10 \%$ de la valeur de la transaction et peut en théorie s'y opposer, en particulier quand les transactions concernent de grandes superficies. Si le patrimoine foncier conserve ses dimensions «traditionnelles", il est, par la force des choses, entré dans le circuit monétaire. Cependant, il n'est cédé qu'en dernier ressort. Les paysans vendent une partie des terres pour faire face à des difficultés de la vie, les plus fréquentes étant la maladie, la mort d'un membre de la famille, ou l'impossibilité de trouver d'autres sources de financement pour assumer les frais de scolarité. Ces charges n'ont rien d'extravagantes, mais elles sont cruciales pour des ménages dont les revenus reposent sur l'agriculture. Une mauvaise année conjuguée avec une maladie peut suffire à motiver la cession d'un lopin de terre.

L'absence de capital est aussi la raison principale de mise en location de champs. Nombre de paysans n'ont pas les moyens de mettre en valeur toute leur exploitation, ou tout simplement ils ne peuvent supporter que les coûts d'une seule culture, et dans ce cas ils choisissent de planter le maïs qui fournit 
l'ugali ${ }^{10}$ consommé quasi quotidiennement. De plus les coûts de production de la pomme de terre et du chou sont très élevés. L'investissement total nécessaire s'élève de 500000 à 1 million de TZS pour cultiver une acre de pomme de terre, à environ 200000 TZS pour une acre de chou. L'épargne est très peu développée : à peine $12 \%$ des personnes interrogées sont membres d'une

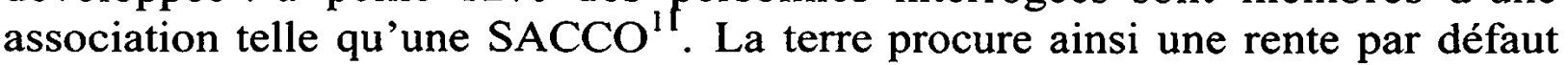
dans les ménages ayant un capital insuffisant pour la mettre eux-mêmes en valeur; elle s'intègre alors dans une stratégie de diversification des ressources.

La transformation de la terre en bien marchand répond aussi aux besoins des acteurs désireux de mettre en production des champs qu'ils ne possèdent pas. Ces individus ont des capitaux très hétérogènes : cela va de l'urbain à faible capital qui souhaite louer à 20000 TZS une acre pour une saison aux business men cherchant à garantir leur approvisionnement pour le marché urbain. Dans le premier cas, la concurrence est faible car le niveau modeste du capital disponible oriente les acteurs vers des secteurs éloignés où le foncier est peu cher ; dans le second elle est par contre très forte. A Ntokela, les agents travaillant pour des donneurs d'ordre cherchent à louer pour plusieurs saisons, afin de cultiver la pomme de terre qui peut être récoltée deux fois dans l'année. Ces transactions sont informelles et fondées sur la confiance. Dans les zones très convoitées - c'est-à-dire facilement accessibles -, les locations se multiplient et s'enchainent sur une même parcelle ce qui peut créer des conflits. Ils éclatent lorsque, par exemple, un locataire veut planter de la pomme de terre alors que le maïs n'a toujours pas, pour des raisons climatiques, été récolté par le locataire précédent ou par le propriétaire. Ces situations extrêmes de multi-locations sont fréquentes dans les hautes altitudes des Uporotos, terres d'élection du solanum tuberosum. A travers ces locations diverses, la terre se banalise et ceux qui louent ont peu de scrupule à tirer le maximum possible d'un sol sollicité à l'extrême. Nombre de locataires ne louent pas les mêmes parcelles d'une année sur l'autre, les baux ne sont pas réguliers ni systématiquement renouvelés.

La terre entre aussi dans la logique commerciale d'hommes d'affaires très éloignés de la montagne, qui cherchent à maximiser leurs profits dans des filières en mettant en concurrence les divers territoires productifs de la

\footnotetext{
${ }^{10}$ L'ugali est le couscous traditionnel fait à partir de maïs. C'est un aliment de base dans de nombreux pays d'Afrique intertropicale appelée foufou au Cameroun, attiéké en Côte d'Ivoire, etc. C'est une pâte réalisée à partir de farines bouillies (maïs ou manioc et parfois les deux mélangés, banane, féculent, tubercules et autres céréales).

${ }^{11}$ SACCO (Saving and Credit Cooperative) : association de micro financement encouragée par le gouvernement dont les formes, les objectifs et les modes de fonctionnement sont multiples mais répondent en principe à l'éthique de participation dans les prises de décision et dans le fonctionnement. La faible adhésion au mouvement coopératif s'explique en partie par la grande méfiance des populations à l'égard de ces structures suite aux nombreuses irrégularités observées dans les années 1980 et par les difficultés à épargner.
} 
Tanzanie. Dans le contexte de libéralisation économique et de dérégulation des filières, la terre en tant que moyen de production garde un caractère incertain, parfois éphémère et dont la « durabilité " n'est pas la priorité des nouveaux acteurs qui la mettent en valeur. Le problème fondamental reste l'accès au capital. Des conditions environnementales favorables, l'ouverture au marché et le contexte libéral n'impliquent pas un enrichissement systématique de la paysannerie montagnarde qui reste cantonnée aux fonctions primaires de la production. La faible capitalisation permet difficilement aux producteurs d'investir le secteur de la commercialisation et par là de renforcer leur capacité de négociation et de décision au sein des filières. Toutes les étapes de mise en vente sont accaparées par les acteurs privés qui prolifèrent et qui sont en concurrence. La carence en capital apparait donc comme le facteur essentiel de la mutation du statut du foncier. La terre semble n'être qu'une variable d'ajustement des stratégies productives marchandes.

Les interrelations entre des acteurs d'origines variées et d'intérêts souvent contradictoires soulignent la complexité des interdépendances entre l'environnement socio-économique global et les contextes locaux. « Le marché (dans le sens de marché physique et d'opportunités de commercialisation) a amené ces changements » disait Mr J.M. élu du village d'Ibililo.

\section{Le statut de la terre : une évolution imparfaite et contradictoire}

La question des marchés fonciers est devenue centrale dans la réflexion sur le développement agricole et rural. "Les marchés de transfert provisoire et définitif de droits sur la terres sont vus comme jouant potentiellement un rôle déterminant dans les processus de développement, en permettant d'améliorer l'allocation des facteurs dans un contexte ou les dotations en facteurs de production et en capacités de gestion sont hétérogènes » [Chauveau \& al. 2006, p. 4]. Cependant, poursuivent les auteurs du projet de recherche CLAIMS, " ...l'accès à la terre sous formes de 'ventes' ne permet pas de considérer comme acquise l'émergence d'un véritable marché foncier " (ibid : 6), qui, pourrait-on ajouter, offrirait un cadre de régulation des tensions et conflits liés à la question foncière. L'enjeu reste bien d'abord l'accès aux ressources multiples à partir desquelles se construisent ou se reconstruisent les trajectoires socio-économiques, individuelles et collectives. Dans ce système de plus en plus complexe, quel rôle joue l'accès au foncier?

\subsection{Les impasses de la patrimonialisation de la terre}

On peut considérer d'abord qu'il y a une difficile mutation du statut de la terre en Afrique du fait d'une modernisation agricole inachevée ou inachevable de par son caractère périphérique et, initialement, de la séparation des secteurs vivrier et marchand [Charlery de la Masselière 2002]. 
La logique du modèle du développement colonial et néocolonial avait consacré le caractère patrimonial de la terre, à un niveau qui se voulait être celui de la famille restreinte. Les enjeux étaient doubles : à la fois faire avancer la frontière agricole des cultures dites commerciales et garantir par là l'assise territoriale des Etats en construction ou en consolidation. Cela a pu se réaliser grâce à un surinvestissement des politiques et des pratiques dans des stratégies foncières paradoxales. Sous l'effet de ces mouvements qui ont affecté à la fois le tissu social et les aires de production, les terroirs se sont profondément fragmentés et diversifiés. Certains se sont marginalisés et repliés sur des structures traditionnelles affaiblies, d'autres se sont ouverts largement sur l'espace plus vaste de l'économie nationale et internationale. On sait que traditionnellement, la terre servait de médiation ou de régulation entre les différents niveaux de la hiérarchie ou de la segmentation sociale. Historiquement, c'est à travers la souplesse des droits coutumiers, quelle que soit leur nature, que se définissaient identités individuelles et collectives, hiérarchies sociales et exercice du pouvoir, dans la profondeur et l'ampleur généalogique des groupes de parenté, dans les rapports de genre, dans la succession des générations ou encore dans les relations de clientèle et de gouvernement. On trouve ici le fondement de la valeur patrimoniale de la terre en Afrique intertropicale, qui a défini le caractère familial des unités de production sur lesquelles les politiques agricoles ont appuyé le développement. La superposition des droits sur la terre reflètent cette histoire sociale et politique complexe.

Comme l'écrit Alain Karsenty [1999, p. 27], «L'ordre moderne [...] affecte l'espace à des usages spécialisés avant de l'affecter à des sujets de droit ». Cette spécialisation a justifié les politiques de sécurisation foncière, supposée à la fois autonomiser le producteur par rapport à ses obligations «traditionnelles » jugées non productives, et garantir son investissement et la maximisation de sa production. Le discours agro-économique sur l'intensification, processus sans cesse inachevé, a progressivement enfermé le producteur dans un champ de servitudes et de contradictions. Devant assurer à la fois la diversité de sa production, garante de sa sécurité alimentaire, la polyvalence de ses activités, garante de sa sécurité économique, et la pluralité de ses capacités d'échange et de redistribution, garante de sa sécurité sociale, le producteur s'est en permanence trouvé en position décalée par rapport à une logique productiviste qui s'est imposée comme modèle dominant. Or l'intensification n'a jamais eu sinon de façon très ponctuelle et éphémère - les moyens de son application. Pour des raisons objectives, ce processus a été réduit à sa simple dimension technique dans laquelle le paysan est entré contre nature quand l'espace se refermait et la terre manquait. Bien plus, la logique intensive a précipité la fixation des droits fonciers et amplifié la fragmentation des unités de production, ôtant toute souplesse à la redistribution des terres, entre les générations en particulier, et démultipliant ainsi les sources de conflits. Déjà en 
1947, au Kilimandjaro et au mont Meru, le rapport Wilson chargé d'étudier la redistribution des terres relevant de la catégorie des Enemy properties, soulignait que l'ouverture des terres nouvelles ne pourrait pas indéfiniment absorber le surplus de la population et que les risques de fragmentation des propriétés poseraient à terme la question de la gestion de l'environnement montagnard. En 1963, dans sa longue introduction à l'ouvrage African Agrarian Systems, Daniel Biebuyck faisait déjà aussi l'inventaire des problèmes liés à l'introduction généralisée et accélérée de la propriété privée. D'un côté était mise en évidence la question de ce que les rapporteurs du programme CLAIMS appellent les faisceaux de droit : "...Il y a beaucoup de flottement lorsqu'il s'agit de définir le contenu de ces droits individuels. On souligne, en particulier, que le droit d'aliéner les biens fonciers n'est pas exercé ou qu'il l'est imparfaitement. On parle de l'aliénation des droits de culture; de l'aliénation du champ, de l'exploitation ou de la palmeraie, de la vente à réméré, mais on hésite à mentionner l'aliénation pure et simple de la terre 》" [Biebuyck 1963, p. 47]. D'un autre, dans les régions à forte densité comme en Afrique de l'Est, étaient mis en lumière les problèmes soulevés par l'appropriation privée sur les structures sociales : problèmes «... de la taille de l'exploitation, du degré de stabilité qu'elle confère, du droit successoral, des conflits particuliers qu'elle crée, de la détermination des mode de preuve pour suivre les contrats conclus » [Ibid , p. 48], pour conclure que «L'introduction de la propriété privée n'est pas nécessairement désirable et son apparition n'est pas inévitable ». [Ibid, p. 50].

On a pu opposer la logique intensive à la consommation minière des ressources productives qui caractérise les fronts pionniers. Mais l'une et l'autre ont produit les mêmes effets de saturation et de cristallisation foncières, alors que la mutation des marchés (des cultures coloniales d'exportation vers le maraîchage pour l'approvisionnement des villes) redéfinit les conditions de la rente et nécessite une mobilité plus importante des terres. A ce blocage foncier, ont répondu de nouvelles formes de crispations identitaires; que ce soit à travers la réactivation de l'autonomie paysanne ou à travers l'émergence de concepts nationalistes ou « ethnicistes», la "rhétorique s'appuie sur une idéologie de l'appartenance au terroir, érigé en référent territorial ultime, et sur la survalorisation du droit du premier occupant " [Janin 2000, p. 793] ainsi que sur l'enfermement des producteurs dans un statut univoque. On peut considérer les années 1990-2000 comme des années de transition, placées sous le signe de l'ambivalence du fait de l'avènement difficile d'un espace citoyen qui s'articule à la fois sur la clientèle ethnique ${ }^{12}$, sur de nouveaux clivages nationalistes et sur les inégalités accrues entre les différentes classes sociales; autant de particularismes qui servent la compétition pour le pouvoir dont les ressources se déplacent vers d'autres secteurs que le secteur proprement

\footnotetext{
12 Dont les identités, si l'on prend l'exemple de l'Afrique de l'Est, sont soit renforcées comme au Kenya, Rwanda et Burundi, soit revitalisées comme en Tanzanie.
} 
agricole ou rural, même si la question foncière reste au cœur des conflits qui ont affecté l'Afrique dans les deux dernières décennies. Le peu d'attention portée aujourd'hui à la question foncière en tant que question paysanne, est significative de l'effacement du sujet paysan qui intéresse de moins en moins les politiques publiques, la recherche ou l'aide au développement. Le foncier est plus une dimension spécifique de la question politique et démocratique, dans le contexte ethnicisé de la compétition pour le pouvoir, qu'une catégorie de l'économie rurale. $\mathrm{Si}$, par ailleurs, les médias internationaux s'intéressent, comme ils ont pu le faire récemment, aux investissements étrangers dans l'agriculture africaine, qui concernent surtout la réaffectation de grands domaines, le paysan resurgit comme simple archétype des inégalités de la globalisation ou comme alibi du développement durable.

\subsection{L'accès au foncier dans la construction d'un "espace de ressource" pluriterritorialisé}

On ne peut plus réduire l'évolution de la logique patrimoniale à la simple gestion des terroirs locaux. Du fait de l'intégration de la paysannerie à la société nationale, les filières de la transmission patrimoniale se sont ouvertes vers d'autres lieux et d'autres activités. La réallocation privilégiée des revenus tirés de l'exploitation agricole, dans la scolarisation des enfants par exemple ou dans des activités urbaines, a fait de la gestion du patrimoine le moteur de la mobilité sociale et géographique. C'est par ce processus de «distanciation », que l'on peut dire que la terre donne lieu à de nouvelles initiatives qui débordent le cadre strictement foncier à l'échelle locale. La crise urbaine et de l'emploi urbain d'un côté, la raréfaction des terres et la baisse des cours des produits de rente d'un autre, ont réactivé ce double jeu - de la terre comme « tenant lieu »de l'identité mais également comme «donnant lieu » à de nouvelles trajectoires-, dans un contexte de compétition accrue pour l'accès aux ressources. D'un point de vue très pragmatique, la législation consacre le simple droit de chacun à se rattacher à une portion de terre, à partir de laquelle il témoigne d'une identité menacée et/ou tente de reconstruire un champ d'activités sociale et économique. L'indétermination demeure sur les modalités concrètes d'intégration à la société globale, qu'elle soit nationale ou relevant d'autres échelles géographiques : le rapport à la terre devient en quelque sorte élémentaire et ne suffit plus à définir le statut du producteur.

Par ailleurs, le dynamisme démographique accélère le renouvellement des générations. "Les générations au sein des familles ne se succèdent pas à proprement parler, mais sont en perpétuelle position de chevauchement [...]. Ce phénomène est source de tensions qui s'expriment ou non sous forme de conflits ouverts, selon que les jeunes générations acceptent ou non les formes de prépartage, les rapports de production ou de circulation du produit, ou les décisions prises par leurs aînés (notamment sur la répartition des droits)... 》 
[Chauveau \& al. 2006, p. 37]. Ce chevauchement des générations accentue les effets de la fragmentation des terres, qui en 1992 pour le Rwanda, nous faisait parler d'implosion spatiale [Charlery de la Masselière 1992]. A cette époque, on avait enregistré des transactions foncières au bénéfice de jeunes exploitants placés en situation de survie, dont l'intekeshwa (parcelle héritée au moment du mariage) était largement insuffisant pour assurer l'approvisionnement du ménage. Ailleurs, comme au pays gusii au Kenya, la diminution des ressources foncières, produit d'un enchainement inéluctable, a pour conséquence l'exclusion progressive des individus dont les droits sont les plus fragiles (femmes, enfants) et la multiplication des conflits fonciers. " A Magenche, un changement progressif des structures ménagères et familiales est en cours depuis plusieurs décennies. Les jeunes hommes gagnent de l'indépendance par rapport à leur père de plus en plus tôt, en lui réclamant une partie de ses terres. Globalement le contrôle des anciens sur la terre et sur les choix matrimoniaux et économique des jeunes s'amenuise. C'est en particulier le cas des jeunes qui attisent les conflits le long de la limite administrative [entre pays gusii et pays masaï] [...]. Cette indépendance des jeunes peut aussi être associée à une acculturation de la société gusii. clé du succès économiques de certains, mais aussi des difficultés sociales et économiques de beaucoup d'autres »[Golaz 2009, p. 217].

On sait que depuis, c'est essentiellement par la migration mais aussi et surtout par la mobilité, les deux en général précaires, entre campagne et ville que jeunes et femmes en particulier essaient de résoudre l'insuffisance des terres qu'ils peuvent exploiter.

Le foncier devient une sorte de fonds de garantie sociale et identitaire. On manque ici de données systématiques pour comprendre et préciser comment le statut de la terre a évolué en ce sens. La propriété - ou selon l'expression consacrée en Afrique, la sécurisation des droits fonciers - ne garantit plus l'accès suffisant aux ressources. La terre entre comme une composante parmi d'autres, sans doute encore indispensable du fait de ce temps de transition et de précarité, d'un système de ressources multiples à géométrie variable. Pour bien comprendre cette place du foncier dans les nouvelles stratégies socioéconomiques des individus, il faut effectivement se demander, si l'on reprend l'expression « faisceau de droits » sur la terre, quels éléments font l'objet de la transaction foncière, de l'individualisation des stratégies ou du champ de solidarités familiales ou extra-familiales. La propriété du sol - qu'elle soit consacrée par la coutume ou dûment enregistrée - conserve la terre dans un registre patrimonial et identitaire de plus en plus anachronique en regard des évolutions et des nécessités économiques contemporaines. Les différents modes de transfert des droits d'usage ou d'exploitation s'adaptent mieux aux contraintes de ces situations «d'entre-deux » hétérogènes et précaires, mais elles ne permettent pas la nécessaire recomposition d'unités d'exploitation économiquement viables. Les inquiétudes demeurent sur le maintien de la 
fertilité ancienne de ces environnements montagnards et sur la cohésion sociale qui l'a entretenue au niveau des unités élémentaires de production.

En conclusion, on peut cependant se demander si, par nécessité et à son insu, l'Afrique n'aurait-elle pas précédé l'âge de la postmodernité ? Evoquant l'écroulement de la propriété comme fondement de la modernité, Jeremy Rifkin précise: "Cela ne veut pas dire que l'âge de l'accès est celui de la disparition pure et simple de la propriété. Bien au contraire, celle-ci reste une réalité centrale de l'activité économique, mais elle est de moins en moins l'objet d'un échange sur un marché. Les pourvoyeurs de l'offre continuent à être propriétaires de biens matériels ou immatériels, mais ils en contrôlent désormais l'accès à travers diverses procédures de location, de leasing, de concession, de droits d'admission, d'adhésion ou d'abonnement qui en définissant l'usage provisoire » [Rifkin 2005, p. 11]. Certes Jeremy Rifkin fait ici référence à des processus du système capitaliste beaucoup plus complexes et à l'échelle globale, mais cela permet peut-être d'inscrire les évolutions foncières de l'Afrique intertropicale dans une logique plus positive : celle d'un nouveau modèle social - sinon d'une révolution sociale dont sur le terrain on sent les prémices - qui marquerait durablement l'histoire des sociétés rurales à l'image du modèle du développement colonial et néocolonial révolu. Paradoxalement, ce nouveau modèle appellerait la mobilisation de la mémoire de l'Afrique sur la façon dont y ont été pendant longtemps gérés les biens communs, gestion dont on a fait une "tragédie ». «Nos idées sur la propriété sont si indissociables des notions traditionnelles de possession et d'exclusion qu'on a du mal à imaginer qu'il existait un droit de propriété plus ancien dont les gens ont joui pendant des siècles : le droit d'accéder à une propriété détenue en commun - par exemple celui de naviguer sur un fleuve, de fourrager dans une forêt locale, de marcher sur un sentier de campagne, de pêcher dans un cours d'eau voisin et de se réunir sur la place publique. Cette idée plus ancienne de la propriété comme droit d'accès et d'inclusion a été progressivement marginalisée à l'époque moderne, où les relations de marché ont dominé la vie et où la propriété privée a été définie comme 'la mesure de l'homme' ". [Rifkin 2012].

\section{Références bibliographiques}

- BIEBUYCK, D. (éd.) (1963) - African Agrarian Systems. International African Institute / Oxford University Press, $407 \mathrm{p}$.

- CHARLERY de la MASSELIERE, B. (1992) - « Le resserrement de l'espace agraire au Rwanda. Les paysans dans la crise ", Etudes rurales, ${ }^{\circ} 125-126$, pp. 99-115

- CHARLERY de la MASSELIERE, B. (2001) - «Perte de la frontière et dérivations identitaires : les paysanneries des hautes terres d'Afrique de l'Est, entre confinement local et transgression des territoires. ", in F. Bart, S. Morin \& J.-C. Salomon (eds), Les montagnes tropicales. Identités, Mutations, Développement. Espaces tropicaux n²16, DYMSET / CRET, pp. 561-577

- CHARLERY de la MASSELIERE, B. (2002) - Paysanneries d'Afrique noire. Entre terre, terroir et territoire. Historiens \& Géographes, $\mathrm{n}^{\circ} 379$ (édition spéciale), pp. 123-132 
- Chauveau, J.-P., COLIN, J.-Ph., JACOB, J.-P., LAVIGNE DELVILlE, Ph. \& LE MEUR, P.-Y. (2006) - Modes d'accès à la terre, marchés fonciers, gouvernance et politiques foncières en Afrique de l'Ouest. Londres, IIED, 91 p., http://pubs.iied.org/pdfs/12528FIIED.pdf

- GOLAZ, V. (2009) - Pression démographiques et changement social au Kenya, IFRA/Karthala, 291 p.

- JANIN, P. (2000) - Crises ivoiriennes et redistribution spatiale de la mobilité : les Baoulé dans la tourmente, Tiers-Monde, vol. 41, n 164, pp. 791-814

- KARSENTY, A. (1999) - «Vers la fin de l'Etat forestier. Appropriation des espaces et partage de la rente foncière au Cameroun », Politique africaine, ${ }^{\circ} 73$, pp. 24-99

- RIFKIN, J. (2005) - L'âge de l'accès. La nouvelle culture du capitalisme, La Découverte, 495 p.

- RIFKIN, J. (2012) - La troisième révolution industrielle, Les liens qui libèrent Editions, $380 \mathrm{p}$.

- SOKONI, C.-H. (2001) - The influence of agricultural marketing reforms on highland farming systems in Tanzania. The case of the Uporoto Highlands, Mbeya Region. Doctorate Thesis. University of Dar es Salaam, 378p.

- WILY, L. A. (2012) - La course mondiale au foncier : quelles implications pour les droits fonciers coutumiers. RRI (Initiative pour les Droits et ressources), Dossier sur l'Etat des lieux de la tenure coutumière en Afrique, janvier 2012, www.rightsandresources.org/ 